\title{
Specific combinations of HLA-DR2 and DR3 class II haplotypes contribute graded risk for disease susceptibility and autoantibodies in human SLE
}

\author{
Robert R Graham ${ }^{1}$, Ward Ortmann ${ }^{2}$, Peter Rodine ${ }^{2}$, Karl Espe $^{2}$, Carl Langefeld ${ }^{3}$,
} Ethan Lange ${ }^{3}$, Adrienne Williams ${ }^{3}$, Stephanie Beck ${ }^{3}$, Chieko Kyogoku ${ }^{2}$, Kathy Moser ${ }^{2}$, Patrick Gaffney ${ }^{2}$, Peter K Gregersen ${ }^{4}$, Lindsey A Criswell ${ }^{5}$, John B Harley ${ }^{6}$ and Timothy W Behrens*,2,7

\footnotetext{
${ }^{1}$ Department of Molecular Biology, Massachusetts General Hospital, Harvard Medical School, Boston, MA, USA; ${ }^{2}$ Department of Rheumatology, Center for Immunology, University of Minnesota Medical School, Minneapolis, MN, USA; ${ }^{3}$ Department of Public Health Sciences, Wake Forest University School of Medicine, Winston-Salem, NC, USA; ${ }^{4}$ Robert $S$ Boas Center for Genomics and Human Genetics, Feinstein Institute for Medical Research, North Shore Long Island Jewish Health System, Manhasset, NY, USA; ${ }^{5}$ Department of Medicine, Division of Rheumatology, Rosalind Russell Medical Research Center for Arthritis, University of California, San Francisco, CA, USA; ${ }^{6}$ Department of Medicine, Oklahoma Medical Research Foundation and University of Oklahoma Health Sciences Center, Oklahoma City, OK, USA
}

The human leukocyte antigen (HLA) Class II antigen presentation alleles DR and DQ are associated with susceptibility to systemic lupus erythematosus (SLE) and the production of lupus-related autoantibodies. Here, we explore the effect of different combinations of Class II risk haplotypes in a large, multi-center collection of 780 SLE families. Haplotypes bearing the DRB1*1501/DQB1*0602 (DR2) and DRB1*0301/ DQB1*0201 (DR3) alleles were present in nearly two-thirds of SLE cases and were significantly associated with disease susceptibility in both family-based and case-control study designs. DR3-containing haplotypes conferred higher risk for disease than DR2, and individual homozygous for DR3 or compound heterozygous for DR3 and DR2 showed the highest risk profile. DR2 haplotypes were also found to be associated with antibodies to the nuclear antigen Sm, and, as previously observed, DR3 genotypes were associated with Ro and La autoantibodies. Interestingly, SLE cases and unaffected family members who were DR2/DR3 compound heterozygotes showed particularly strong risk of developing antibodies to Ro, and were enriched for La and Sm. These data provide convincing evidence that particular combinations of HLA Class II DR2 and DR3 haplotypes are key determinants of autoantibody production and disease susceptibility in human SLE.

European Journal of Human Genetics (2007) 15, 823-830; doi:10.1038/sj.ejhg.5201827; published online 4 April 2007

Keywords: systemic lupus erythematosus; human leukocyte antigen; association study

\footnotetext{
*Correspondence: Dr TW Behrens, Immunology, Tissue Growth \& Repair, Genentech, Inc, Exploratory Clinical Development, 1 DNA Way, S San Francisco, CA 94080, USA. Tel: 650467 4319/243 8269; Fax: 650225 8221; E-mail: behrens.tim@gene.com

${ }^{7}$ Current address: Genentech, Inc., South San Francisco, CA, USA Received 4 April 2006; revised 12 January 2007; accepted 27 February 2007; published online 4 April 2007
}

Introduction

Systemic lupus erythematosus (SLE, MIM :152700) is a systemic, inflammatory autoimmune disease that affects $\sim 275000$ people in the US (0.1\% population prevalence), ${ }^{1}$ and exhibits a strong gender bias (9:1, female/male ratio). Lupus is clinically heterogeneous, and patients often 
experience a progressing cycle of disease flares and remissions. Nearly half of cases will manifest severe complications of the disease, which may include glomerulonephritis and compromised renal function, and/or neurologic symptoms such as seizures, psychiatric symptoms, peripheral neuropathies or stroke. Despite advances in treatment, the mortality rate of SLE patients remains three times that of matched controls. ${ }^{2}$

SLE is thought to result from complex interactions among environmental, hormonal, and genetic factors. ${ }^{3-6}$ A genetic component for SLE is well-established and is supported by twin studies, the familial aggregation of disease, and the identification of several confirmed SLE risk genes. ${ }^{7-11}$ The first genetic association described for SLE was with the human leukocyte antigen (HLA) region. ${ }^{9}$ The HLA region is contained within a $3.6 \mathrm{Mb}$ interval located on human chromosome $6 \mathrm{p} 21.3$ that encodes over 200 genes, many with known roles in immunity. ${ }^{12,13}$ The telomeric Class I region contains the ubiquitously expressed HLA-A, -B, and -C genes, which present antigenic peptides to $\mathrm{CD} 8+\mathrm{T}$ cells. The centromeric Class II region contains the highly polymorphic HLA-DR, -DQ and -DP genes. These molecules are expressed at high levels on antigen-presenting cells, such as dendritic cells and B cells and display peptides for CD $4+$ helper T cells. The Class III region lies between the Class I and II regions and contains many immune-related genes, including the cytokines tumor necrosis factor- $\alpha$ and lymphotoxin- $\alpha$ and the complement components C2, C4 and Factor B.

In addition to the importance of the HLA for transplantation tolerance, ${ }^{14}$ genes within the region are also major contributors to the risk for autoimmunity. ${ }^{15}$ For instance, Class I alleles are associated with psoriasis (Cw6) and ankylosing spondylitis (B27), whereas HLA Class II alleles are associated with rheumatoid arthritis (DRB1*0401 DR4), celiac disease (DQA $\left.1{ }^{*} 0501, \mathrm{DQB} 1{ }^{*} 0201\right)$, multiple sclerosis (DRB1*1501 - DR2), IgA deficiency (DRB1*0301 DR3), autoimmune thyroid disease (DR3), type 1 diabetes mellitus (DR3, DR4) and SLE (DR2, DR3).

Antibodies specific for nucleic acids and nuclear autoantigens are a hallmark of SLE, and are involved at many levels in disease pathogenesis. ${ }^{16,17}$ At least $4 \%$ of individuals in the general population show elevated serum titers of anti-nuclear antibodies (ANAs) in the absence of rheumatic disease, and the presence of ANAs may increase the risk for SLE as much as 40 -fold. ${ }^{18}$ Of interest, nuclear autoantibodies such as the Ro and La RNA-binding proteins can be detected in the serum of many SLE cases well before the onset of clinical symptoms. ${ }^{18}$

Association studies in SLE populations of European descent have generally concluded that haplotypes bearing DR3 are associated with SLE susceptibility, whereas haplotypes bearing DR2 appear to be more weakly associated (reviewed in references ${ }^{19,20}$ ). In a recent study, we demonstrated that the disease-associated HLA-DR2 containing haplotype is localized to a $500 \mathrm{~kb}$ interval that includes the DR and DQ genes and two genes of unknown function. ${ }^{21}$ HLA-DR3 haplotypes showed strong linkage disequilibrium, often extending $>1 \mathrm{Mb}$ into the Class III and I regions, such that localization of the primary genetic effect(s) on this haplotype was difficult. Anti-La and antiRo antibodies have consistently demonstrated association with the DR3 Class II haplotypes, ${ }^{22,23}$ whereas the association of antibodies to other HLA alleles remains unclear.

In the present study, we genotyped HLA Class II alleles in two large, independent replication cohorts, and examined the data together with previous typing performed in 283 MN SLE families (total of 780 pedigrees). We also examined serum autoantibody profiles in 584 SLE cases for association to HLA genotypes. These data provide support for the hypothesis that DR2- and DR3-containing haplotypes, alone and in combinations, are potent contributors to genetic risk for autoantibodies and disease-susceptibility in SLE.

\section{Materials and methods Family collections}

We studied a multi-center US Caucasian SLE family collection of 780 pedigrees, composed of 1158 SLE cases and 1545 other family members (Table 1). The clinical features of disease and demographics of these families are described elsewhere. ${ }^{24-30}$ The combined collection consists of 283 sib-pair and trio pedigrees collected at the University of Minnesota (MN), 146 multiplex families from the Oklahoma Medical Research Foundation (OMRF) provided by the Lupus Multiplex Registry and Repository (AR12253), and 351 trio pedigrees collected at the University of California-San Francisco (UCSF). These studies were approved by the Human Subject Institutional Review Boards at each institution, and informed consent was obtained from all subjects.

\section{Genotyping}

Class II haplotypes were determined by typing three HLA region microsatellite markers (D6S2666, D6S2665,

Table 1 Composition of three independent caucasian SLE family collections

\begin{tabular}{lcccr}
\hline & \multicolumn{4}{c}{ SLE cohort } \\
& MN & \\
& OMRF $^{\mathrm{b}}$ & UCSF & All \\
\hline Multiplex/sib-pair pedigrees & 164 & 146 & - & 310 \\
Trio pedigrees & 119 & - & 351 & 470 \\
Total pedigrees & 283 & 146 & 351 & 780 \\
Affected individuals & 471 & 325 & 362 & 1158 \\
Unaffected individuals & 466 & 427 & 652 & 1545 \\
Total individuals & 937 & 752 & 1014 & 2703 \\
\hline
\end{tabular}

aniversity of Minnesota.

${ }^{\mathrm{b}}$ Oklahoma Medical Research Foundation.

'University of California - San Francisco. 
D6S2446) that flank the DR locus and are in tight linkage disequilibrium. ${ }^{21}$ Our previous studies demonstrated that these markers tag Class II haplotypes with high specificity and fidelity. ${ }^{21,31}$ The markers were genotyped in all 2703 individuals at the University of Minnesota, as described. ${ }^{21}$ Detailed information regarding primer sequences and locations are presented in Supplementary Table S1, and allele frequency distributions are shown in Supplementary Table S2. Microsatellite marker haplotypes for the pedigrees and controls were constructed using standard parameters of PHASE v2.1. ${ }^{32}$ Samples $(N=44)$ with $>50 \%$ missing data or where phase could not be confidently assigned with a probability $<50 \%(N=260)$ were excluded from the analysis.

To verify further the ability of haplotypes constructed from the D6S2666/2665/2446 microsatellites to faithfully represent underlying HLA Class II haplotypes, we genotyped the D6S2666/2665/2446 markers in 902 Caucasian control individuals from the New York Cancer Project (NYCP) collection, ${ }^{33}$ for whom DRB1 allele status was available as determined by oligotyping. ${ }^{34}$ The specificity of the microsatellite haplotypes as surrogates for all of the common DRB1 alleles averaged $99.8 \%$. The ability of the microsatellite haplotypes to predict correctly the DRB1 allele (as determined by oligotyping) averaged 99.0\% and for the microsatellite haplotypes bearing DRB1*1501/ DQB1*0602 (DR2), DRB1*0301/DQB1*0201 (DR3) and DRB1*0801/DQB1*0402 (DR8) the positive predictive value was $96.7,100$ and $100 \%$ respectively.

In the NYCP controls, the microsatellite analysis identified a few rare marker combinations for HLA- DRB ${ }^{*} 02$ and DRB ${ }^{*} 03$ (as defined by low-resolution oligotyping) (Supplementary Table S3). The rare HLA-DRB1*02 haplotypes and two of the HLA-DRB $1{ }^{*} 03$ haplotypes showed no evidence or trend of being enriched in SLE cases, and were excluded from the current analysis. One of the rare HLADRB ${ }^{*} 03$ associated marker haplotypes was found at an allele frequency of $1.72 \%$ of cases and $0.89 \%$ of controls $(P=0.05)$. This variant haplotype shares the identical alleles at D6S2666 and D6S2665 with the common HLADRB ${ }^{*} 0302$ haplotype, but differs by three repeat units at D6S2446 and likely represents the DRB1*0302 allele. Although showing borderline evidence for association, this uncommon DR3 microsatellite variant was excluded from the current analysis. The conclusions of this present study were not different when this haplotype was included in the analysis (data not shown).

\section{Autoantibody measurements}

Autoantibody data from 584 SLE patients (from 274 independent pedigrees of the OMRF and MN collections) and 533 unaffected relatives were used in the analyses. Precipitating (high-titer) levels of Abs reactive with ENAs included anti-Ro/SSA, anti-La/SSB, anti-Sm, anti-nRNP and anti-ribosomal $\mathrm{P}$ protein and were detected by
Ouchterlony assays. ${ }^{35,36}$ These autoantibodies were dichotomously classified as being 'present' or 'absent'. Abs reactive with anti-dsDNA were measured by Crithidiae lucilliae assays (Protrac Industries, Kerrville, TX, USA). All individuals with detectable anti-dsDNA Abs at a titer of 1:120 were considered positive.

\section{Analysis \\ Family-based association analysis To avoid the poten-} tial bias of selecting a single case from multiplex pedigrees, a single trio or, if only one parent was available, a single dyad from each pedigree was randomly selected. The random trio/dyad selection was repeated 10000 times. The TDT/S-TDT 1.1 program $^{37}$ (a valid test of association in trio and dyad pedigrees) was then applied to each of the pedigree files and an average of the transmissions/ non-transmissions for each run was calculated. The nominal $P$ value was calculated for the $\chi^{2}$ generated from $2 \times 2$ contingency tables.

To assess further the statistical significance of the results, permutation testing was employed. Using Haploview v3.2 (http://www.broad.mit.edu/mpg/haploview/), the transmitted/untransmitted status of each haplotype was randomly permutated for 100000 iterations and the best $\chi^{2}$ value generated for each permutated dataset was recorded. The number of times the permutated $\chi^{2}$ value exceeded the nominal $\chi^{2}$ value was divided by the number of iterations (100000) to generate the permutated $P$ value.

The pedigree disequilibrium test (PDT) and genotype PDT (genoPDT) were performed using PDT v5.1 with default settings. ${ }^{38,39}$ The PDT and TDT were performed using single microsatellite markers and microsatellite haplotypes. For several of the analyses, the microsatellite haplotypes were coded such that the 'non-risk' HLA haplotypes were grouped into a single allele (DRX) and tested for association relative to the HLA 'risk' haplotypes.

Autoantibody association analysis SLE cases or unaffected family members were stratified by HLA genotype, and the number and frequency of autoantibody positive samples was determined for each HLA genotype. The frequency of autoantibodies (Ro, La, Sm, P, RNP, the presence of $>1$ ENA and dsDNA) in individuals carrying an HLA 'risk' haplotype was compared with the frequency of antibodies in individuals not carrying an HLA 'risk' haplotype (DRX/DRX). Only one of 461 unaffected family members tested positive for dsDNA antibodies, therefore dsDNA antibodies were not analyzed in these individuals. The significance of the differences in autoantibody frequencies between individuals carrying the risk haplotypes and individuals not carrying a risk haplotype (DRX/DRX) was assessed by calculating the $P$ value after correcting for familial correlation by using the empirical estimate of the variance. $^{40}$ 


\section{Results}

Family-based association testing of HLA class II risk haplotypes in SLE

We examined the association of HLA Class II haplotypes in a replication cohort, comprised of two independent SLE family collections from OMRF and UCSF (total $N=497$ ) (Table 2). Application of the Transmission Disequilbrium Test (TDT) to the dataset confirmed that DR2- $(P=0.02)$ and DR3-containing $\left(P=2.1 \times 10^{-4}\right)$ haplotypes were associated with SLE in the replication cohort, and in the combined collection (Table 2). The DR8 haplotype was also over-transmitted, but was relatively rare in this collection and the association failed to reach statistical significance. Similar significance levels were obtained by permutation of the transmission and non-transmission status of parental chromosomes (Table 2). No other HLA class II haplotypes demonstrated a positive association with SLE (data not shown). The PDT, a global test of association appropriate for complex pedigrees, also showed highly significant transmission distortion at the Class II region; global $P=3 \times 10^{-5}$ for the 497 replication pedigrees, and global $P=1 \times 10^{-12}$ in the combined dataset of 780 pedigrees. In addition, the allele and genotype frequencies of the HLA Class II haplotypes in 780 unrelated SLE cases selected the pedigrees and 902 Caucasian controls from the $\mathrm{NYCP}^{33}$ database were examined to estimate the effect of these alleles relative to the general population (Supplementary Table S4). From these analyses using both family-based and case-control designs, we conclude that haplotypes containing DR2, DR3 and possibly DR8 are associated with susceptibility to SLE.

\section{Genotypic risk of HLA class II haplotypes in SLE}

To address the combinatorial effects of HLA risk haplotypes and model of inheritance, we analyzed the transmission distortion of genotypes in these families using the genotype-PDT (genoPDT). ${ }^{39}$ In the genoPDT, instead of measuring the transmission patterns of single alleles within a trio as in the TDT or PDT, the transmission of the genotype is measured. Of the four parental alleles in each trio, the two transmitted alleles comprise the transmitted genotype, whereas the two non-transmitted parental alleles are considered the non-transmitted genotype. While the allele-based PDT is a more powerful test under simple additive models, the genoPDT has more power under dominant and recessive models. ${ }^{39}$

Applying the genoPDT to this dataset revealed a transmitted/non-transmitted (T/NT) ratio of 1.3 for DR2/ DRX (where $\mathrm{X}$ is any non-DR2 or non-DR3 haplotype) $(P=0.04)$ and 1.4 for DR3/DRX genotypes $(P=0.03)$ (Table 3). A significantly higher T/NT ratio was observed for DR3/DR3 (T/NT =2.1, $P=0.03$ ) and for DR2/DR3 (T/ $\mathrm{NT}=2.3, P=0.0001)$ genotypes, consistent with a dose effect of the DR3 haplotype and an increased risk in DR2/ DR3 compound heterozygotes.

\section{Autoantibody associations with class II genotypes}

We did not observe significant associations between HLA class II status and ACR criteria for SLE in the 780 SLE pedigrees (data not shown), consistent with previous studies (Harley et al., 1998a). We next examined whether the identified HLA haplotypes were associated with autoantibodies in this family collection. Sera of 584 SLE cases

Table 2 Transmission disequilibrium test for HLA Class II haplotypes in 780 SLE families

\begin{tabular}{|c|c|c|c|c|c|c|c|}
\hline & Haplotype & $T$ & $N T$ & $T / N T$ & $\chi^{2}$ & Nominal $P$ & Permuted $P^{\mathrm{a}}$ \\
\hline Original cohort ${ }^{\mathrm{b}}$ (283 pedigrees) & $\begin{array}{l}\text { DR2 } \\
\text { DR3 } \\
\text { DR8 } \\
\text { non-risk }\end{array}$ & $\begin{array}{l}69 \\
59 \\
14 \\
53\end{array}$ & $\begin{array}{r}37 \\
30 \\
7 \\
121\end{array}$ & $\begin{array}{l}1.86 \\
1.97 \\
2.00 \\
0.44\end{array}$ & $\begin{array}{r}9.7 \\
5.4 \\
2.3 \\
26.6\end{array}$ & $\begin{array}{c}1.9 \times 10^{-3} \\
2.1 \times 10^{-3} \\
\mathrm{NS} \\
2.5 \times 10^{-7}\end{array}$ & $\begin{array}{c}0.0022 \\
0.001 \\
\text { NS } \\
<0.00001\end{array}$ \\
\hline Replication cohort ${ }^{\mathrm{C}}$ (497 pedigrees) & $\begin{array}{l}\text { DR2 } \\
\text { DR3 } \\
\text { DR8 } \\
\text { non-risk }\end{array}$ & $\begin{array}{l}87 \\
89 \\
13 \\
80\end{array}$ & $\begin{array}{r}59 \\
46 \\
10 \\
155\end{array}$ & $\begin{array}{l}1.47 \\
1.93 \\
1.30 \\
0.52\end{array}$ & $\begin{array}{r}5.4 \\
13.7 \\
0.4 \\
23.9\end{array}$ & $\begin{array}{c}0.02 \\
2.1 \times 10^{-4} \\
\text { NS } \\
1.0 \times 10^{-6}\end{array}$ & $\begin{array}{c}0.058 \\
0.0003 \\
\text { NS } \\
0.00002\end{array}$ \\
\hline 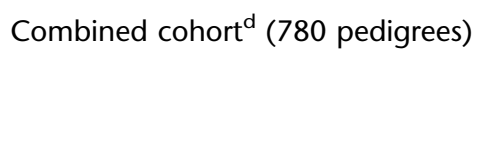 & $\begin{array}{l}\text { DR2 } \\
\text { DR3 } \\
\text { DR8 } \\
\text { non-risk }\end{array}$ & $\begin{array}{r}157 \\
149 \\
27 \\
135\end{array}$ & $\begin{array}{r}96 \\
76 \\
17 \\
279\end{array}$ & $\begin{array}{l}1.64 \\
1.96 \\
1.59 \\
0.48\end{array}$ & $\begin{array}{r}14.7 \\
23.7 \\
2.3 \\
50.1\end{array}$ & $\begin{array}{c}1.3 \times 10^{-4} \\
1.1 \times 10^{-6} \\
\text { NS } \\
1.5 \times 10^{-12}\end{array}$ & $\begin{array}{c}0.0003 \\
<0.00001 \\
\text { NS } \\
<0.00001\end{array}$ \\
\hline
\end{tabular}

DR2, DRB1*1501/DQB1*0602; DR3, DRB1*0301/DQB1*0201; DR8, DRB1*0801/DQB1*0402. Haplotypes containing the indicated DRB1/DQB1 alleles were determined by microsatellite genotypes for the markers D6S2666/2665/2446 as described in the text.

T, transmitted haplotype; NS, not significant, $P>0.05$; NT, non-transmitted haplotype.

a Permuted P, 100000 random permutations of transmitted and non-transmitted chromosome status, as described in Materials and methods.

${ }^{\mathrm{b}} \mathrm{MN}$ families.

${ }^{\mathrm{C} O M R F}$ and UCSF families.

${ }^{\mathrm{d} M N}$, OMRF and UCSF families. 
obtained from 273 independent OMRF and MN pedigrees were assayed for the presence of autoantibodies to the following extractable nuclear antigens (ENAs): Ro/SSA, La/ SSB, RNP, P, and Sm. The frequency of affected individuals positive for ENAs carrying a given HLA risk genotype was compared with the frequency of affected individuals positive for ENAs who did not carry an HLA risk haplotype (Table 4).

In general, individuals carrying the DR2 and DR3 haplotypes were more likely to be positive for antibodies to several of these nuclear antigens (Supplementary Table S6). Compared with DRX/DRX cases, DR2/DRX individuals were enriched for $\mathrm{Sm}$ antibodies $(P=0.0022)$, whereas DR3/DRX individuals had higher frequencies of antibodies to La $(P=0.0005)$ and Ro $(P=0.0003)$. DR2/DR3 compound heterozygotes were enriched for antibodies to $\mathrm{La}$ and $\mathrm{Sm}$, and were three times more likely than DRX/DRX cases to exhibit antibodies to Ro $\left(P=2 \times 10^{-6}\right)$. Nearly $60 \%$ of DR2/DR3 individuals were positive for at least one antibody to an extractable nuclear antigen, compared to $28.7 \%$ of SLE cases not carrying a risk haplotype $(P=0.0047)$.
Data for anti-dsDNA antibodies, available for 449 cases from 208 pedigrees, were similarly analyzed. In DR2/DR3 compound heterozygotes, 22 of 35 individuals (62.9\%) were positive for anti-dsDNA antibodies, compared with only $28.4 \%$ of DRX/DRX individuals (50 of 176 , $P=8.3 \times 10^{-5}$ ). No other HLA genotype showed significant association with dsDNA antibodies.

The relatively low numbers of DR2 $(N=13)$ and DR3 $(N=19)$ homozygote individuals in this dataset reduced the power to assess significance of the results. However, the DR2/DR2 group was consistent with the trends observed in DR2/DRX cases (significant association of Ro and Sm), whereas the DR3/DR3 genotype showed strong association with Sm, but not with Ro or $\mathrm{La}$, and a trend towards significance for association with anti-dsDNA antibodies.

\section{Discussion}

We have studied the contribution of HLA Class II haplotypes to disease susceptibility and specific autoantibody production using a large multi-center collection of SLE families (780 pedigrees) and a large control cohort

Table 3 Genotype-pedigree disequilibrium test in 517 SLE trios ${ }^{a}$

\begin{tabular}{|c|c|c|c|c|c|}
\hline Genotype $^{\mathrm{b}}$ & $T$ & $N T$ & $T / N T$ & Z score & $P$ \\
\hline DR2/DRX & 135 & 104 & 1.3 & 2.0 & 0.04 \\
\hline DR3/DRX & 100 & 71 & 1.4 & 2.2 & 0.03 \\
\hline DR2/DR2 & 12 & 13 & 0.9 & -0.2 & NS \\
\hline DR3/DR3 & 21 & 10 & 2.1 & 2.2 & 0.03 \\
\hline DR2/DR3 & 51 & 22 & 2.3 & 3.5 & 0.0001 \\
\hline DRX/DRX & 198 & 297 & 0.7 & -5.6 & $2.0 \times 10^{-8}$ \\
\hline
\end{tabular}

${ }^{a} 517$ unrelated complete trios (genotype data available for both parents and affected offspring), corresponding to 1034 total transmitted and untransmitted genotypes.

${ }^{\mathrm{b}} \mathrm{DR} 2, \mathrm{DRB} 1{ }^{*} 1501 / \mathrm{DQB1}{ }^{* 0602}$; DR3, DRB1*0301/DQB1*0201; DRX, haplotype other than DR2 or DR3. Haplotypes containing the indicated DRB1/ DQB1 alleles were determined by microsatellite genotypes for the markers D6S2666/2665/2446 as described in the text; T, transmitted genotype; NT, non-transmitted genotype; NS, not significant.

Table 4 Autoantibody associations with HLA Class II genotypes

\begin{tabular}{|c|c|c|c|c|c|c|c|c|c|c|c|}
\hline & \multirow[t]{2}{*}{$\begin{array}{c}D R X / D R X \\
N=230^{\mathrm{a}} \%(N)\end{array}$} & \multicolumn{2}{|c|}{$\begin{array}{c}D R 2 / D R X \\
N=159\end{array}$} & \multicolumn{2}{|c|}{$\begin{array}{c}D R 3 / D R X \\
N=121\end{array}$} & \multicolumn{2}{|c|}{$\begin{array}{c}D R 2 / D R 3 \\
N=42\end{array}$} & \multicolumn{2}{|c|}{$\begin{array}{c}D R 2 / D R 2 \\
N=13\end{array}$} & \multicolumn{2}{|c|}{$\begin{array}{c}D R 3 / D R 3 \\
N=19\end{array}$} \\
\hline & & $\%(N)$ & P-value & $\%(N)$ & P-value & $\%(N)$ & P-value & $\%(N)$ & $P$ value & $\%(N)$ & $P$ value \\
\hline La & 5 & 6 & $N S^{d}$ & 18212 & & 1436 & & & & $0(0)$ & - \\
\hline & & & & (4) & & & 0.0 & (5) & & $0(0)$ & - \\
\hline $\mathrm{Sm}$ & 1.7 (4) & 9.4 (15) & 0.0022 & $4.1(5)$ & $\mathrm{N}$ & 7.1 (3) & 0.0731 & $15.4(2)$ & 0.0066 & 15.8 (3) & 0.0028 \\
\hline $\mathrm{D}$ & 0.9 (2) & $0.6(1)$ & NS & $0(0)$ & ${ }^{f}$ & $0(0)$ & - & 7.7 (1) & 0.0784 & $0(0)$ & - \\
\hline $\mathrm{RN}$ & $15.2(35)$ & $15.7(25)$ & $\mathrm{N}$ & 9.9 (12) & NS & $9.5(4)$ & NS & $23.1(3)$ & NS & $15.8(3)$ & NS \\
\hline ANY ENA & $\begin{array}{c}28.7 \\
N=176\end{array}$ & $\begin{array}{c}37.1 \\
\mathrm{~N}=119\end{array}$ & NS & $\begin{array}{l}43(52) \\
\mathrm{N}=90\end{array}$ & 0.0035 & $\begin{array}{c}59.5(25) \\
\mathrm{N}=35\end{array}$ & 0.0047 & $\begin{array}{c}53.9(7) \\
\mathrm{N}=12\end{array}$ & NS & $\begin{array}{c}15.8(3) \\
N=17\end{array}$ & NS \\
\hline $\mathrm{ds} D N A^{\mathrm{c}}$ & $28.4(50)$ & $31.9(38)$ & NS & $26.7(24)$ & NS & $62.9(22)$ & 0.000083 & $25(3)$ & NS & $47.1(8)$ & NS \\
\hline
\end{tabular}

DRX, haplotypes other than DR2 or DR3; DR2, DRB1*1501/DQB1*0602; DR3, DRB1*0301/DQB1*0201. Haplotypes containing the indicated DRB1/ DQB1 alleles were determined by microsatellite genotypes for the markers D6S2666/2665/2446 as described in text.

${ }^{a} N$, Number of SLE individuals with indicated genotypes;

${ }^{b} P$ value, familial correlation was accounted for by using a sandwich estimator of the variance and exchangeable correlation.

Individuals positive for dsDNA antibodies at serum dilutions of 1:120.

dNot significant $(P>0.1)$

e Individuals positive for 1 or more extractable nuclear antigens (ENA), defined as La, Ro, Sm, P, or RNP.

${ }^{f}$ Could not be calculated. 
$(N=902)$. Using a variety of different analytical approaches, a remarkably clear and consistent picture emerges, with the key findings as follows: (1) using family-based association testing, we have replicated earlier work suggesting that DR2- and DR3-containing Class II haplotypes confer significant risk for the development of SLE in Caucasians; (2) we show that DR3/DR3 and DR2/DR3 are particularly strong risk genotypes for SLE susceptibility; (3) we find that a single dose of DR2 elevates the probability of autoantibodies to Sm, whereas a single dose of DR3 is associated with Ro and La autoantibodies; and (4) we identify compound heterozygosity for DR2/DR3 as a common (7.5\% of SLE cases) and particularly potent risk genotype for antinuclear antibodies, and show that this genotype also predisposes to autoantibodies in unaffected family members of SLE cases. Together, these data provide important new insights into the role of specific combinations of Class II risk haplotypes in SLE disease susceptibility and autoantibody targeting.

We have used an approach to genotype Class II alleles that is based on the typing of three highly polymorphic microsatellites that flank the DR and DQ loci, rather than genotyping DR by oligotyping or other sequence-based methods. We verified that this method is a highly reliable alternative to direct oligotyping by analyzing these microsatellites in a large control cohort where DR oligotyping was performed in parallel (Rodine et al., in preparation).

Class II genotyping by the microsatellite haplotype method is highly sensitive and specific, indicating that the lengths of the measured dinucleotide-repeat markers has remained very stable over population history. An important advantage of microsatellite-based assignment of DR is the cost savings. These three microsatellite markers can be multiplexed into a single PCR, and performed for less than \$1.00/genotype, which is $\sim 100$-fold savings over oligotyping. Since our data suggests that the microsatellite genotyping method can be applied to all of the common Caucasian DR alleles (Rodine et al., in preparation), we believe that this method can be applied widely and in a cost-effective fashion to a broad range of diseases. It is important to note that the current studies have focused on the application of this typing method in Caucasians, and further work will be required to validate this method for other ethnic groups.

Compound heterozygosity and homozygosity for HLA risk alleles have been shown to confer an increased risk for several autoimmune diseases. Homozygosity for DRB $1{ }^{*} 0401$ and heterozygosity for DRB $1{ }^{*} 0401 /$ DRB $1{ }^{*} 0404$ is associated with an increased risk for rheumatoid arthritis. ${ }^{41}$ Heterozygosity for DR3/DR4 is strongly enriched in type 1 diabetes, whereas DR2 homozygosity shows strong association in multiple sclerosis. ${ }^{42,43}$ Of interest, early studies suggested the possibility of genetic complementation between DR2/DQ6 and DR3/DQ2 haplotypes in susceptibility to lupus, as well as to Sjogren's syndrome, a disease related to SLE characterized by a high incidence of antinuclear antibodies. ${ }^{44-47}$

How can compound heterozygosity for DR2/DR3 or homozygosity for DR3 contribute to the lupus phenotype? Heterozygosity of HLA antigen presentation molecules confers improved resistance to infection, presumably because of the larger and more diverse antigen repertoire that can be presented by heterozygous individuals. ${ }^{48,49}$ Similarly, individual heterozygous for the HLA Class II alleles associated with SLE may have an increased probability of displaying self-reactive antigens. Recent data suggest that breaks in tolerance to viral proteins (eg, EBNA1 protein of EBV) may be associated with subsequent cross-reactive autoimmunity to RNA-binding proteins such as Ro and Sm. ${ }^{50,51}$ One hypothesis that warrants consideration is that the relevant viral- or self-antigens in SLE may be restricted by the disease-associated DR2 and DR3 Class II alleles (and/or their respective DQ alleles) for presentation to $\mathrm{CD} 4+\mathrm{T}$ cells, thereby increasing the probability that autoimmune responses might be initiated and propagated. An alternate hypothesis is that CD4 + and $\mathrm{CD} 25+\mathrm{T}$ regulatory cells, which are important for suppressing autoimmune diseases, ${ }^{52}$ may undergo inefficient positive selection on the SLE risk Class II alleles. The low background population prevalence of antibodies to ENAs $(\sim 1 \%)$ and the progressive appearance of these antibodies before the onset of clinical symptoms suggests that loss of immunologic tolerance to these antigens marks an important transition in the road to overt disease. ${ }^{18}$ The possibility that DR2 or DR3 Class II molecules might be able to present peptides from the relevant EBV gene products and the Ro, La and Sm autoantigens should be explored.

Our previous studies visualizing recombinations on the ancestral haplotypes carrying DR2 allowed us to localize the major genetic-risk region on this haplotype to an $\sim 500 \mathrm{~kb}$ region containing both DRB1 and DQB1. ${ }^{21}$ The only other genes in this interval are two genes of unknown function - chromosome 6 open reading frame 10 (C6orf10 formerly known as testis specific basic protein), and butyrophilin-like family member II (BTNL2) - together with DRA (invariant), DRB3 and DRB5 (additional $\beta$ chain genes present on the DRB $1{ }^{*} 0301$ and DRB1*1501 haplotypes, respectively), and DQA (a different allele for each of the risk haplotypes). Further studies are required to determine whether the genetic effects on this haplotype are limited to the Class II DRB1 and/or DQB1 genes, or include these other tightly linked genes.

The extensive linkage disequilibrium on the DRB1*0301 haplotype severely limits the ability to localize the genetic effect on this haplotype. The increased risk of the DR3 haplotype relative to the DR2 haplotype makes it tempting to hypothesize that multiple genetic effects reside on this haplotype. For example, a high-expressing TNF- $\alpha$ allele and C4 'null' alleles, which have been suggested previously as 
risk factors for SLE, ${ }^{53,54}$ are both carried on the extended A1/B8/DR3 haplotype. At this point, caution is warranted before assigning risk to any individual gene on the DRB ${ }^{*} 0301$ haplotype, given the extensive disequilibrium observed. Typing of a dense SNP map across the entire $\mathrm{HLA}^{55}$ in DR3 + SLE cases and controls, may allow us to tease out additional genetic contributions to SLE present on this haplotype.

\section{Acknowledgements}

We gratefully acknowledge the many patients, families and physicians who have cooperated for the purposes of genetic studies in SLE. This work was supported by grants, contracts and fellowships from the National Institute of Arthritis, Musculoskeletal and Skin Diseases, the National Institute of Allergy and Infectious Disease, the Minnesota Lupus Foundation, the Mary Kirkland Center for Lupus Research, and the Alliance for Lupus Research. These studies were performed in part at the General Clinical Research Center, Moffitt Hospital, University of California, San Francisco, with funds provided by the National Center for Research Resources (5 M01 RR00079, US Public Health Service) and at the Oklahoma Medical Research Foundation with grants awarded to JBH (NIH grants: AR12253, AI24717, AR42460, AR048094, RR024013, AI32584, and AR048940).

\section{References}

1 Hochberg MC, Perlmutter DL, Medsger TA et al: Prevalence of selfreported physician-diagnosed systemic lupus erythematosus in the USA. Lupus 1995; 4: 454-456.

2 Borchers AT, Keen CL, Shoenfeld Y, Gershwin ME: Surviving the butterfly and the wolf: mortality trends in systemic lupus erythematosus. Autoimmun Rev 2004; 3: 423-453.

3 Hochberg MC: The epidemiology of systemic lupus erythematosus; in: Wallace DJ, Hahn BH (eds): Dubois' Lupus Erythematosus. Williams and Wilkins: Baltimore, 1997; 49-65.

4 Wakeland EK, Liu K, Graham RR, Behrens TW: Delineating the genetic basis of systemic lupus erythematosus. Immunity 2001; 15: $397-408$

5 Nath SK, Kilpatrick J, Harley JB: Genetics of human systemic lupus erythematosus: the emerging picture. Curr Opin Immunol 2004; 16: 794-800.

6 Gaffney PM, Moser KL, Graham RR, Behrens TW: Recent advances in the genetics of systemic lupus erythematosus. Rheum Dis Clin North Am 2002; 28: 111-126.

7 Deapen DM, Escalante A, Weinrib L, Horwitz DA, Mack TM: A revised estimate of twin concordance in systemic lupus erythematosus. Arthritis Rheum 1992; 35: 311-318.

8 Hochberg M, Florsheim P, Scott J, Arnett FC: Familial aggregation of systemic lupus erythematosus. Arthritis Rheum 1985; 28: 523.

9 Goldberg MA, Arnett FC, Bias WB, Shulman LE: Histocompatibility antigens in systemic lupus erythematosus. Arthritis Rheum 1976; 19: 129-132.

10 Prokunina L, Castillejo-Lopez C, Oberg F et al: A regulatory polymorphism in PDCD1 is associated with susceptibility to systemic lupus erythematosus in humans. Nat Genet 2002; 32: 666-669.

11 Kyogoku C, Langefeld CD, Ortmann WA et al: Genetic association of the R620W polymorphism of protein tyrosine phosphatase PTPN22 with human SLE. Am J Hum Genet 2004; 75: 504-507.

12 Aguado B, Bahram S, Beck S et al: Complete sequence and gene map of a human major histocompatibility complex. Nature 1999; 401: $921-923$.

13 Rhodes DA, Trowsdale J: Genetics and molecular genetics of the MHC. Rev Immunogenet 1999; 1: 21-31.
14 Snell GD: Methods for the study of histocompatibility genes. J Genet 1948; 49: 87.

15 Vyse TJ, Todd JA: Genetic analysis of autoimmune disease. Cell 1996; 85: 311-318.

16 Reichlin M, Harley JB: Antibodies to Ro/SSA and La/SSB; In: Wallace DJ, Hahn BH (eds): Dubois' Lupus Erythematosus. Lippincott Williams \& Wilkins: Philadelphia, PA, 2002, pp 467-480.

17 Reveille JD: Predictive value of autoantibodies for activity of systemic lupus erythematosus. Lupus 2004; 13: 290-297.

18 Arbuckle MR, McClain MT, Rubertone MV et al: Development of autoantibodies before the clinical onset of systemic lupus erythematosus. N Engl J Med 2003; 349: 1526-1533.

19 Harley JB, Moser KL, Gaffney PM, Behrens TW: The genetics of human systemic lupus erythematosus. Curr Opin Immunol 1998; 10: 690-696.

20 Tan FK, Arnett FC: The genetics of lupus. Curr Opin Rheumatol 1998; 10: 399-408.

21 Graham RR, Ortmann WA, Langefeld CD et al: Visualizing human leukocyte antigen class II risk haplotypes in human systemic lupus erythematosus. Am J Hum Genet 2002; 71: 543-553.

22 Meyer O, Hauptmann G, Tappeiner G, Ochs HD, Mascart-Lemone F: Genetic deficiency of $\mathrm{C} 4, \mathrm{C} 2$ or $\mathrm{C} 1 \mathrm{q}$ and lupus syndromes. Association with anti-Ro (SS-A) antibodies. Clin Exp Immunol 1985; 62: 678-684.

23 Hartung K, Ehrfeld H, Lakomek HJ et al: The genetic basis of Ro and La antibody formation in systemic lupus erythematosus. Results of a multicenter study. The SLE study group. Rheumatol Int 1992; 11: 243-249.

24 Gaffney PM, Kearns GM, Shark KB et al: A genome-wide search for susceptibility genes in human systemic lupus erythematosus sibpair families. Proc Natl Acad Sci USA 1998; 95: 14875-14879.

25 Gaffney PM, Ortmann WA, Selby SA et al: Genome screening in human systemic lupus erythematosus: results from a second Minnesota cohort and combined analyses of 187 sib-pair families. Am J Hum Genet 2000; 66: 547-556.

26 Graham RR, Langefeld CD, Gaffney PM et al: Genetic linkage and transmission disequilibrium of marker haplotypes at chromosome 1q41 in human systemic lupus erythematosus. Arthritis Res 2001; 3: 299-305.

27 Moser KL, Neas BR, Salmon JE et al: Genome scan of human systemic lupus erythematosus: evidence for linkage on chromosome 1q in African-American pedigrees. Proc Natl Acad Sci USA 1998; 95: 14869-14874

28 Moser KL, Gray-McGuire C, Kelly J et al: Confirmation of genetic linkage between human systemic lupus erythematosus and chromosome 1q41. Arthritis Rheum 1999; 42: 1902-1907.

29 Seligman VA, Suarez C, Lum R et al: The Fcgamma receptor IIIA$158 \mathrm{~F}$ allele is a major risk factor for the development of lupus nephritis among Caucasians but not non-Caucasians. Arthritis Rheum 2001; 44: 618-625.

30 Parsa A, Peden E, Lum RF et al: Association of angiotensinconverting enzyme polymorphisms with systemic lupus erythematosus and nephritis: analysis of 644 SLE families. Genes Immun 2002; S42-S46.

31 Jawaheer D, Li W, Graham RR et al: Dissecting the genetic complexity of the association between human leukocyte antigens and rheumatoid arthritis. Am J Hum Genet 2002; 71: 585-594.

32 Stephens M, Donnelly P: A comparison of bayesian methods for haplotype reconstruction from population genotype data. Am J Hum Genet 2003; 73: 1162-1169.

33 Mitchell MK, Gregersen PK, Johnson S, Parsons R, Vlahov D: The New York Cancer Project: rationale, organization, design, and baseline characteristics. J Urban Health 2004; 81: 301-310.

34 Lee HS, Li W, Lee A et al: Microsatellite typing for DRB1 alleles: application to the analysis of HLA associations with rheumatoid arthritis. Genes Immun 2006; 7: 533-543.

35 Clark G, Reichlin M, Tomasi Jr TB: Characterization of a soluble cytoplasmic antigen reactive with sera from patients with systemic lupus erythmatosus. J Immunol 1969; 102: 117-122. 
36 Ramos PS, Kelly JA, Gray-McGuire C et al: Familial aggregation and linkage analysis of autoantibody traits in pedigrees multiplex for systemic lupus erythematosus. Genes Immunol 2006; 7: 417-432.

37 Spielman RS, Ewens WJ: A sibship test for linkage in the presence of association: the sib transmission/disequilibrium test. Am J Hum Genet 1998; 62: 450-458.

38 Martin ER, Monks SA, Warren LL, Kaplan NL: A test for linkage and association in general pedigrees: the pedigree disequilibrium test. Am J Hum Genet 2000; 67: 146-154.

39 Martin ER, Bass MP, Gilbert JR, Pericak-Vance MA, Hauser ER: Genotype-based association test for general pedigrees: the genotype-PDT. Genet Epidemiol 2003; 25: 203-213.

40 Zeger SL, Liang KY: Longitudinal data analysis for discrete and continuous outcomes. Biometrics 1986; 42: 121-130.

41 Hall FC, Weeks DE, Camilleri JP et al: Influence of the HLA-DRB1 locus on susceptibility and severity in rheumatoid arthritis. Qjm 1996; 89: 821-829.

42 Noble JA, Valdes AM, Cook M, Klitz W, Thomson G, Erlich HA: The role of HLA class II genes in insulin-dependent diabetes mellitus: molecular analysis of 180 Caucasian, multiplex families. Am J Hum Genet 1996; 59: 1134-1148.

43 Barcellos LF, Oksenberg JR, Begovich AB et al: HLA-DR2 dose effect on susceptibility to multiple sclerosis and influence on disease course. Am J Hum Genet 2003; 72: 710-716.

44 Harley JB, Alexander EL, Bias WB et al: Anti-Ro (SS-A) and anti-La (SS-B) in patients with Sjogren's syndrome. Arthritis Rheum 1986; 29: 196-206.

45 Hamilton RG, Harley JB, Bias WB et al: Two Ro (SS-A) autoantibody responses in systemic lupus erythematosus. Correlation of HLA-DR/DQ specificities with quantitative expression of Ro (SS-A) autoantibody. Arthritis Rheum 1988; 31: 496-505.
46 Harley JB, Sestak AL, Willis LG, Fu SM, Hansen JA, Reichlin M: A model for disease heterogeneity in systemic lupus erythematosus. Relationships between histocompatibility antigens, autoantibodies and lymphopenia or renal disease. Arthritis Rheum 1989; 32: 826-836.

47 Fujisaku A, Frank MB, Neas B, Reichlin M, Harley JB: HLA-DQ gene complementation and other histocompatibility relationships in man with the anti-Ro/SSA autoantibody response of systemic lupus erythematosus. J Clin Invest 1990; 86: 606-611.

48 Hughes AL, Yeager M: Natural selection at major histocompatibility complex loci of vertebrates. Annu Rev Genet 1998; 32: 415-435.

49 Parham P, Ohta T: Population biology of antigen presentation by MHC class I molecules. Science 1996; 272: 67-74.

50 McClain MT, Heinlen LD, Dennis GJ, Roebuck J, Harley JB, James JA: Early events in lupus humoral autoimmunity suggest initiation through molecular mimicry. Nat Med 2005; 11: 85-89.

51 McClain MT, Poole B, Bruner BF, Kaufman KM, Harley JB, James JA: An altered immune response to Epstein-Barr virus nuclear antigen-1 (EBNA-1) in pediatric systemic lupus erythematosus. Arthrit Rheum 2006; 54: 360-368.

52 Paust S, Cantor H: Regulatory T cells and autoimmune disease. Immunol Rev 2005; 204: 195-207.

53 Arnett FC: The genetic basis of lupus erythematosus; in: Wallace DJ, Hahn BH (eds): Dubois' Lupus Erythematosus. Williams \& Wilkins: Baltimore, 1997, pp 77-117.

54 Harley JB, Moser KL, Gaffney PM, Behrens TW: The genetics of human systemic lupus erythematosus. Curr Opin Immunol 1998; 10: 690-696.

55 Walsh EC, Mather KA, Schaffner SF et al: An integrated haplotype map of the human major histocompatibility complex. Am J Hum Genet 2003; 73: 580-590.

Supplementary Information accompanies the paper on European Journal of Human Genetics website (http://www.nature.com/ejhg) 